\title{
Endoscopic facelift of the frontal and temporal areas in multiple planes
}

\author{
Xiaogen $\underline{\mathrm{Hu}}{ }^{1}$, MD, Haihuan $\underline{M a}^{1}$, MD, Zhiqiang $\underline{X u e^{1}}$, MD, Huijie $\underline{\mathrm{Qi}}{ }^{1}$, MD, Bo $\underline{C h e n^{1}}$, MD
}

\begin{abstract}
INTRODUCTION The detachment planes used in endoscopic facelifts play an important role in determining the results of facial rejuvenation. In this study, we introduced the use of multiple detachment planes for endoscopic facelifts of the frontal and temporal areas, and examined its outcome.

METHODS This study included 47 patients ( 38 female, 9 male) who requested frontal and temporal facelifts from January 2009 to January 2014. The technique of dissection in multiple planes was used for all 47 patients. In this technique, the frontal dissection was first carried out in the subgaleal plane, before being changed to the subperiosteal plane about $2 \mathrm{~cm}$ above the eyebrow line. Temporal dissection was carried out in both the subcutaneous and subgaleal planes. After detachment, frontal and temporal fixations were achieved using nonabsorbable sutures, and the incisions were closed. During follow-up (ranging from 6-24 months after surgery), the patients were shown their pre- and postoperative images, and asked to rate their satisfaction with the procedure. Complications encountered were documented.

RESULTS All 47 patients had complete recovery without any serious complications. The patient satisfaction rate was 93.6\%. Minor complications included dimpling at the suture site, asymmetry, overcorrection, transitory paralysis, late oedema, haematoma, infection, scarring and hair loss. These complications resolved spontaneously and were negligible after complete recovery.

CONCLUSION Dissection in multiple planes is valuable in frontal and temporal endoscopic facelifts. It may be worthwhile to introduce the use of this technique in frontal and temporal facelifts, as it may lead to improved outcomes.
\end{abstract}

Keywords: dissection, endoscope, frontal and temporal facelift, multiple planes

\section{INTRODUCTION}

Endoscopic facelifts in the frontal and temporal areas were first introduced to clinical practice about two decades ago. ${ }^{(1)}$ The technique used for this procedure has been developed with time. The detachment technique used during frontal and temporal facelift plays an important role in the outcome of facial rejuvenation. The use of alternative detachment planes for endoscopic facelifts of the frontal and temporal areas has been reported. ${ }^{(2,3)}$ In the present study, we introduced the use of multiple detachment planes for endoscopic facelifts of the frontal and temporal areas, and reported our five-year experience with the use of this technique. The use of multiple detachment planes was introduced with the hope of achieving better results.

\section{METHODS}

This study included 47 patients (38 female, 9 male) who requested frontal and temporal facelifts from January 2009 to January 2014. The mean age of the patients was 37 (range 34-48) years. The follow-up period ranged from six months to 24 months. During follow-up, the patients were shown their pre- and postoperative images, and asked to rate their satisfaction with the procedure. They could rate the improvement as 'less than expected', 'as expected' or 'greater than expected'. If the patient rated the improvement as 'less than expected', it was taken to mean that the procedure was unsatisfactory to the patient. If the patient rated the improvement as 'as expected' or 'greater than expected', it was taken to mean that the procedure was satisfactory to the patient. Any complications encountered perioperatively were reported and documented.

For the procedure, the patient was placed under intravenous sedation in the supine position. Nerve blocks were performed by infiltrating the supraorbital and supratrochlear nerve areas with $1 \%$ lidocaine and epinephrine $(1: 200,000)$. Two sagittal frontal incisions (about $1 \mathrm{~cm}$ behind the frontal hairline) and two coronal incisions (about $4 \mathrm{~cm}$ behind the temporal hairline) were made. Each incision was about $2 \mathrm{~cm}$ long. Using the frontal incisions as access points, frontal detachment was first carried out in the subgaleal plane up to about $2 \mathrm{~cm}$ above the eyebrow line. Thereafter, the detachment plane was changed from the subgaleal plane to the subperiosteal plane, up to the supraorbital nerve; the supraorbital adhesions and periorbital septum were detached (Figs. 1a \& b).

Before detachment, the temporal area was infiltrated subcutaneously with a solution containing saline, epinephrine and lidocaine (i.e. $0.1 \mathrm{mg}$ of epinephrine and $200 \mathrm{mg}$ of lidocaine per $100 \mathrm{cc}$ of saline). The average amount of infiltrated solution used was 50-100 cc for one side of the temporal area.

Temporal detachment was carried out in both the subcutaneous and subgaleal planes. Subcutaneous detachment was done downward to the zygomatic arc level, forward to the lateral canthus and upward to the ridge of the temporal crest. Subcutaneous dissection was initiated carefully so that the hair

${ }^{1}$ Plastic Surgery Department, China Japan Friendship Hospital, Beijing, China

Correspondence: Dr Xiaogen Hu, Consultant, Plastic Surgery Department, China Japan Friendship Hospital, 2 Yinghua E St, Chaoyang Qu, Beijing 100029, China. huxiaogeng78@sina.com 

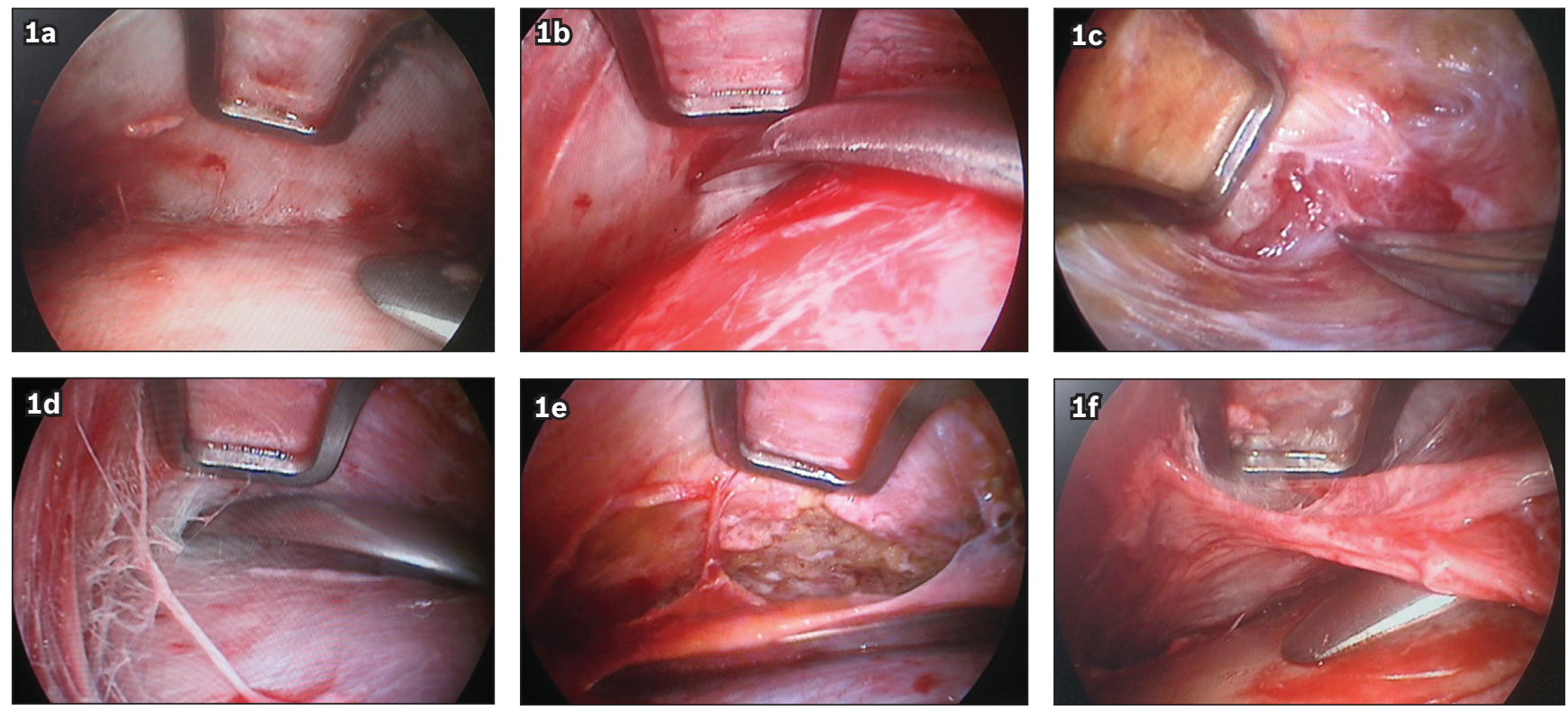

Fig. 1 Endoscopic images show (a) detachment in the subgaleal plane; (b) detachment from the subgaleal plane to subperiosteal plane, about 2 cm above the eyebrow line; (c) subcutaneous detachment in the temporal area; (d) subgaleal undermining of the temporal area; (e) elevation of the scalp flap and fascia flap during temporal dissection; and (f) frontal dissection being connected to the temporal subgaleal detachment at the temporal crest.

follicles were well protected from damage. During detachment, the subcutaneous fat was clearly seen (Fig. 1c). Haemostasis was performed meticulously and immediately after the dissection was completed. In the subgaleal plane, blunt dissection was utilised (Fig. 1d); detachment was done upward to the temporal crest, forward to the orbital rim and downward to the zygomatic arc. After subgaleal detachment, the fascia flap was elevated (Fig. 1e). Finally, the frontal detachment was connected to the temporal dissection in the subgaleal plane at the temporal crest (Fig. 1f).

After frontal and temporal detachment, two frontal fixation stitches were made in the forehead. The frontal composite flap was advanced and anchored to the bony tunnel made in the frontal bone. Temporal fascia fixation was also used on each side of the temple. The elevated temporal fascia flap was advanced and fixed to the deep temporal fascia at the level of the temporal incision. All the fixation sutures were nonabsorbable. Finally, the correct position of the eyebrow for each side was verified by adjusting the tension of the fixation until symmetry was achieved. The excess scalp flap was trimmed and the incisions were closed with tension-free sutures. No drain was needed. The operative areas were dressed with a traditional bandage, which was applied for seven days. All the scalp sutures and dressings were removed one week after the operation.

\section{RESULTS}

All 47 patients had complete recovery without any serious complications. The patient satisfaction rate was 93.6\% (44/47). The representative cases are shown in Figs. 2-4. We encountered some minor complications, including dimpling at the suture site, asymmetry, overcorrection, transitory paralysis, late oedema, haematoma, infection, scarring and hair loss (Table I). These complications resolved spontaneously and were negligible after complete recovery.

\section{DISCUSSION}

The use of endoscopy for facial cosmetic surgery was first introduced in the 1990s. (1) After its introduction, many anatomical studies were conducted on the frontal and temporal fascial layers, and on the relationship of these fascial layers with the facial nerves; these studies were done to gather information that would facilitate endoscopic rejuvenation in those areas. ${ }^{(2,3)}$ Endoscopic facelift in the frontal and temporal areas has been practised clinically for more than a decade. ${ }^{(1,4,5)}$ The accumulation of experience in this procedure has boosted the use of endoscopy in facelifts.

The plane of detachment that was used for frontal and temporal facelifts plays a very important role in ensuring the success of the facelift. Traditionally, dissection is carried out in a single plane, both in the frontal and temporal areas. ${ }^{(6,7)}$ In early frontal and temporal facelifts, the detachment in the frontal area was carried out in the simple subcutaneous or areolar plane. Subsequently, more consideration was given to protect the supraorbital and supratrochlear nerves, and the dissection plane was thus changed to the subperiosteal plane. Each detachment plane has its advantages and disadvantages. Subcutaneous dissection helps to mobilise the frontal skin flap, which enables effective reduction of frontal wrinkles; however, it also increases the risk of injury to the supraorbital and supratrochlear nerves. On the other hand, subperiosteal dissection can protect the aforementioned nerves from damage and has good haemostasis, but results in limited lifting (i.e. it is less effective in reducing frontal wrinkles). This is because dissection in the simple subperiosteal plane decreases the mobility of the frontal flap due to the firm texture of the periosteum. In the present study, the frontal detachment was first carried out in the subgaleal plane, up to about $2 \mathrm{~cm}$ above the eyebrow line. The detachment plane was then changed to the subperiosteal plane. This method of detachment avoided the risks associated with dissection in a 

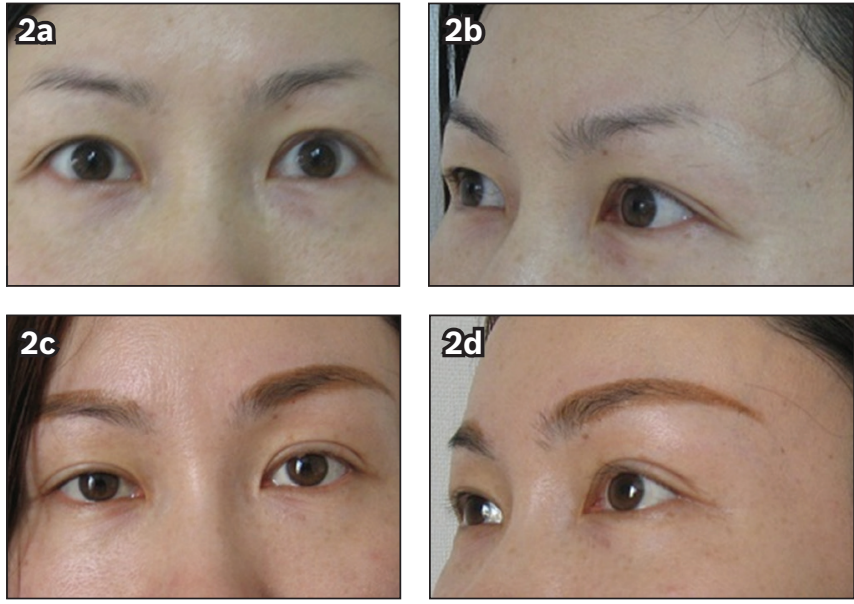

Fig. 2 Preoperative photographs show the (a) frontal and (b) oblique views of a 36-year-old woman who presented with ptosis of the upper eyelid and lateral canthus. Postoperative photographs show the (c) frontal and (d) oblique views of the patient eight months after endoscopic facelift of the frontal and temporal areas.
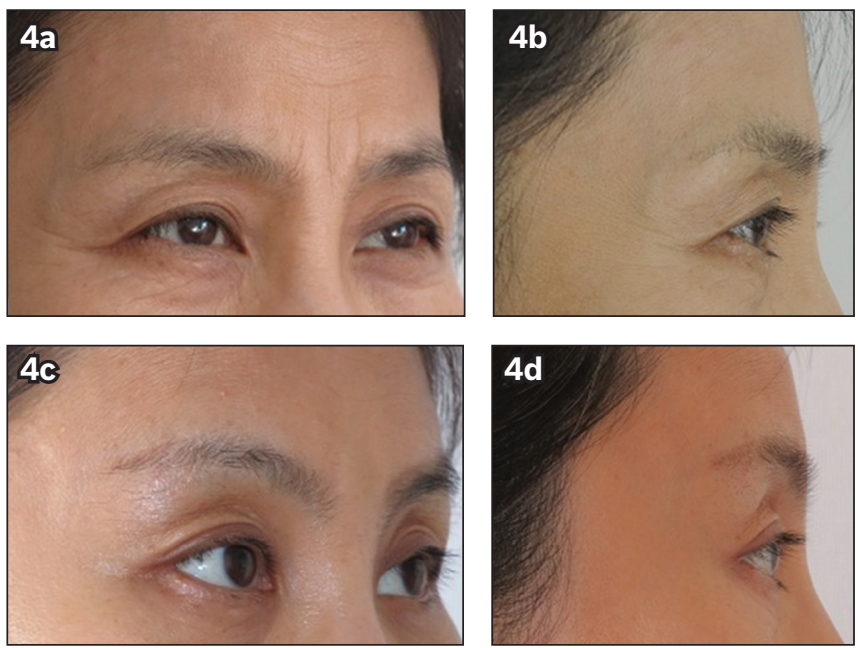

Fig. 4 Preoperative photographs show the (a) oblique and (b) lateral views of a 47-year-old woman who presented with marked brow ptosis, and periorbital and frontal wrinkles. Postoperative photographs show the (c) oblique and (d) lateral views of the patient six months after endoscopic facelift of the frontal and temporal areas.

single plane, and the benefits of this method were evidenced among all 47 patients.

Detachment in the temporal area was carried out in the subcutaneous plane in early frontal and temporal facelifts. Dissection in the subcutaneous plane can result in significant alleviation of wrinkles and elevation of the lateral eyebrow. Hair follicles are also well protected from damage during subcutaneous dissection of the temporal area. Consequently, hair loss is avoided; this is important for patients who are already afflicted with alopecia. ${ }^{(8)}$ However, the effects of subcutaneous dissection are not lasting, and a second temporal facelift is often needed for sustained results. ${ }^{(9)}$ These days, detachment in the temporal area is more commonly performed in the subgaleal plane. ${ }^{(10,11)}$ However, the rejuvenation effect is compromised when dissection is carried out in only one plane. This is because the ageing speed of the skin and temporal galea is different. Therefore, dissection in multiple planes in the temporal area can increase the rejuvenation effect. In the present study, after complete two-layer detachment
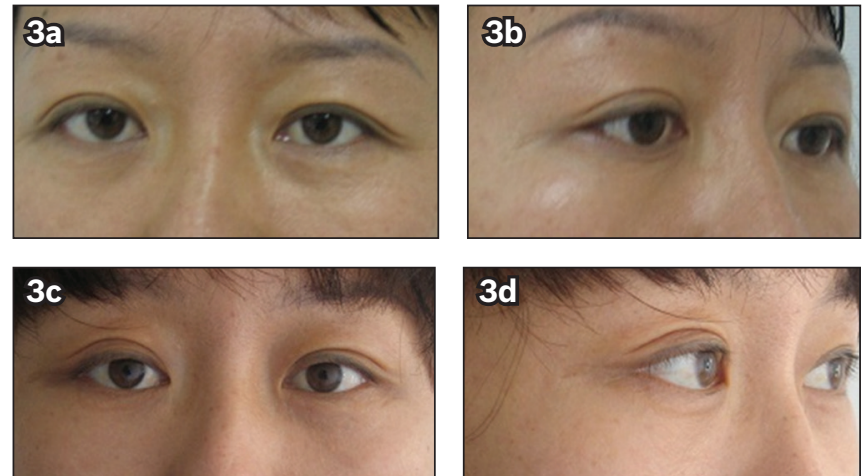

Fig. 3 Preoperative photographs show the (a) frontal and (b) oblique views of a 36-year-old woman who presented with moderate ptosis of the upper eyelid and lateral canthus. Postoperative photographs show the (c) frontal and (d) oblique views of the patient seven months after endoscopic facelift of the frontal and temporal areas.

Table I. Postoperative complications observed $(n=47)$.

\begin{tabular}{lc}
\hline Complication & No. (\%) \\
\hline Dimpling at suture site & $3(6.4)$ \\
Asymmetry & $4(8.5)$ \\
Overcorrection & $2(4.3)$ \\
Transitory paralysis & $1(2.1)$ \\
Haematoma & $2(4.3)$ \\
Oedema or prolonged ecchymosis & $1(2.1)$ \\
Obvious scars & $2(4.3)$ \\
Complaint of hair loss & $2(4.3)$ \\
\hline
\end{tabular}

in the temporal area, the frontal branch of the facial nerve was embedded in the temporal fascia flap. As excessive traction of the fascia flap during fixation might cause damage to the frontal branch of the facial nerve, ${ }^{(12)}$ advancement of the fascia flap with proper tension must be kept in mind during traction. As attention was paid to this detail in our practice, injury of the facial nerve was rare in the present study.

Several authors have recommended the use of multiple dissection planes for facelifts of the frontal and temporal areas. ${ }^{(13,14)}$ Fogli explained the benefits of using different dissection planes for temporal facelifts in an article published in 2003. ${ }^{(15)}$ The dissection planes used by Fogli included the subperiosteal, subgaleal and subcutaneous planes; dissection was performed using the different planes to create a temporal composite flap. The technique used by Fogli can only be employed by surgeons who have a clear understanding of the anatomical structures of the temporal area and are skilled in temporal lifts. In the present study, the dissection was carried out under endoscopic vision; the facial nerve and other important tissues were well protected. Furthermore, the simple two-layer dissection that was used for the temporal area can be easily understood and conducted.

Endoscopic facelifts are popular because the incisions made are small and recovery is relatively quick. Done through a minimal-access incision, endoscopic facelifts have proven to be useful in selected cases. ${ }^{(16)}$ They may replace traditional coronal facelifts for middle-aged persons who request for frontal and temporal facelifts, as traditional coronal facelifts are characterised by considerably extensive trauma and slow recovery. However, for 
older patients who may have more severe laxity and sloppy skin in the frontal and temporal areas, the use of the coronal incision method is still recommended, as it may yield better results.

We recognise that the present study has several limitations. This is a small retrospective, descriptive study, and the duration of the postoperative follow-up was also limited.

To conclude, the use of multiple planes in endoscope-assisted frontal and temporal facelifts may be valuable for selected patients. Additional clinical studies, longer follow-up intervals and greater data sharing are needed to validate the efficacy of this treatment method.

\section{REFERENCES}

1. Isse NG. Endoscopic facial rejuvenation: endoforehead, the functional lift. Case reports. Aesthetic Plast Surg 1994; 18:21-9.

2. Campiglio GL, Candiani P. Anatomical study on the temporal fascial layers and their relationships with the facial nerve. Aesthetic Plast Surg 1997; 21:69-74.

3. Moss CJ, Mendelson BC, Taylor GI. Surgical anatomy of the ligamentous attachments in the temple and periorbital regions. Plast Reconstr Surg 2000; 105:1475-90.

4. Raspaldo H. [Video-assisted endoscopic lifting: development]. Rev
Laryngol Otol Rhinol (Bord) 1997; 118:57-64. French.

5. Keller GS, Mashkevich G. Endoscopic forehead and brow lift. Facial Plast Surg 2009; 25:222-33

6. Maloney BP, Schiebelhoffer J. Minimal-incision endoscopic face-lift. Arch Facial Plast Surg 2000; 2:274-8.

7. Jacono AA, Parikh SS. The minimal access deep plane extended vertical facelift. Aesthet Surg J 2011; 31:874-90.

8. Shipchandler TZ, Sultan B, Byrne PJ. Endoscopic forehead lift in patients with male pattern baldness. Am J Otolaryngol 2012; 33:519-22.

9. Bidros RS, Salazar-Reyes H, Friedman JD. Subcutaneous temporal browlift under local anesthesia: a useful technique for periorbital rejuvenation. Aesthet Surg J 2010; 30:783-8.

10. Hunt JA, Byrd HS. The deep temporal lift: a multiplanar lateral brow, temporal, and upper face lift. Plast Reconstr Surg 2002; 110:1793-6.

11. Erel E, Wong M, Chester D, Vijh V. Modified Fogli temporal lift. J Plast Reconstr Aesthet Surg 2011; 64:693-5.

12. Pitanguy I, Machado BH. Facial rejuvenation surgery: a retrospective study of 8788 cases. Aesthet Surg J 2012; 32:393-412.

13. Baker SR. Triplane rhytidectomy. Combining the best of all worlds. Arch Otolaryngol Head Neck Surg 1997; 123:1167-72.

14. Tobin HA, Cuzalina A, Tharanon W, Sinn DP. The biplane facelift: an opportunistic approach. J Oral Maxillofac Surg 2000; 58:76-85.

15. Fogli AL. Temporal lift by galeapexy: a review of 270 cases. Aesthetic Plast Surg 2003; 27:159-65.

16. Hu XG, Ma HH, Fu XZ, et al. Minimally traumatic midface lift approach for patients in their early 30s and 40s. J Chin Med Assoc 2010; 73:487-91. 